\title{
BANCO DE SEMENTES COMO INDICADOR DE RESTAURAÇÃO EM SISTEMAS AGROFLORESTAIS MULTIESTRATIFICADOS NO SUDOESTE DE MATO GROSSO DO SUL, BRASIL ${ }^{1}$
}

\author{
Murilo Moressi², Milton Parron Padovan ${ }^{3}$ e Zefa Valdivina Pereira ${ }^{4}$
}

\begin{abstract}
RESUMO - Este estudo teve por objetivo avaliar a composição do banco de sementes como indicador de restauração em quatro módulos de sistemas agroflorestais multiestratificados, com diferentes idades e em remanescente florestal nativo no Município de Amambai, região Sudoeste do Estado de Mato Grosso do Sul. As coletas de solo foram realizadas em dezembro de 2012 e consistiram em 15 amostras de $20 \mathrm{~cm}$ x $20 \mathrm{~cm}$, a $5 \mathrm{~cm}$ de profundidade, distribuídas ao acaso, em cada módulo do Sistema Agroflorestal e testemunha (mata), com descarte da serapilheira, totalizando 75 amostras. As amostras foram alocadas em estufa e acondicionadas em bandejas plásticas de $3 \mathrm{~L}$, agrupadas lado a lado por área de coleta. O monitoramento das plântulas ocorreu durante 180 dias, com irrigação diária. A maioria das espécies foi de herbáceas (73\%), representando as arbóreas 14,2\%. Entre as arbóreas, três se destacam: Trema micrantha (L.) Blume, Cecropia pachystachya Trecul e Solanum paniculatum L. Em relação à densidade de sementes viáveis no solo, ocorreu predomínio de espécies herbáceas em todas as áreas, e os valores não apresentaram diminuição em relação ao aumento em idade das áreas; entretanto, os menores valores foram encontrados na área de Mata. A densidade de sementes de espécies arbóreas aumentou conforme o avanço em idade das áreas, sugerindo o avanço sucessional dos sistemas agroflorestais multiestratificados estudados, de forma semelhante ao que ocorre em florestas em estádio secundário de sucessão, evidenciando sua capacidade em restaurar áreas degradadas.
\end{abstract}

Palavras-chave: Agroecossistemas sustentáveis; Regeneração florestal: Sucessão natural.

\section{SEED BANK AS INDICATOR OF RESTORATION IN MULTISTRATA AGROFORESTRY SYSTEMSIN SOUTHWESTERN OF MATO GROSSO DO SUL, BRAZIL}

\begin{abstract}
This studyaimed to evaluatethe composition of theseed bankas indicator ofrestorationin fourmodules of multistrataagroforestry systems, with differentages, andin a remaining native forestin the Municipality ofAmambai, southwestern regionof the state of Mato Grossodo Sul. Thesoilsampleswere takenin December 2012and consisted in 15 samples of $20 \mathrm{~cm} \times 20 \mathrm{~cm}, 5 \mathrm{~cm}$ depth, randomly distributedin eachmodule of agroforestry systemandcontrol (forest area), with disposal of the leaf litter, totaling 75samples. The sampleswere placedin a greenhouse, in plastic trays of $3 \mathrm{~L}$ and groupedsideby side, by collection area. The monitoringwas made for180 days with daily irrigation.Most specieswereherbaceous(73\%), the trees representing14.2\%. Among thetree species, three stand out: Trema micrantha (L.) Blume, Cecropia pachystachya Trécul, and Solanum paniculatum L. Inrelation to the densityof viable seedsin the soil, there was a predominanceof herbaceous speciesin all areas, and the values for these speciesshowed nodecreasein relation toincreasing age of the areas; however,the lowest valueswere foundin theforest area. The seed density of tree species increased according to the age of the areas, suggesting successional advancement of themultistrata agroforestry systems studied, similar to what occurs in forests in secondarysuccessional stage, demonstrating its ability to restore degraded areas.
\end{abstract}

Keywords: Sustainable agroecosystem; Forest regeneration; Natural succession.

\footnotetext{
${ }^{1}$ Recebido em 29.10.2013 aceito para publicação em 08.10.2014.

${ }^{2}$ Universidade Federal da Grande Dourados, Programa de Pós-Graduação em Biologia Geral/Bioprospecção, Dourados, MS

- Brasil. E-mail: <murilomoressi@yahoo.com.br>.

${ }^{3}$ Universidade Federal da Grande Dourados, Faculdade de Ciências Biológicas e Ambientais, Dourados, MS - Brasil. E-mail: <zefaPereira@ufgd.edu.br>.

${ }^{4}$ Empresa Brasileira de Pesquisa Agropecuária, Embrapa Agropecuária Oeste. Dourados, MS - Brasil. E-mail: <padovan@cpao.embrapa.br>.
} 


\section{INTRODUÇÃO}

O banco de sementes é formado pela produção local e por sementes alóctones, sendo essas prontamente viáveis, ou em estado de dormência, presentes na serapilheira ou no solo (HARPER, 1977; FENNER, 1985). Segundo Simpson et al. (1989), este sistema pode ser transitório, com sementes viáveis apenas por um ano, ou persistentes, com sementes que permanecem viáveis no solo por mais de um ano. Essa permanência representa uma reserva do potencial genético acumulado e está intrinsecamente relacionada à resiliência do hábitat, que se traduz pela capacidade de um ecossistema se recuperar após um distúrbio, considerando suas funções e processos ecológicos.

O conhecimento básico do banco de sementes permite que se possa predizer sobre o potencial florístico existente no processo de sucessão; dessa forma, pode ser um bom preditor dos processos de regeneração de ecossistemas (RODRIGUES; GANDOLFI, 1998), uma vez que, através da sua avaliação, é possível definir estratégias para conduzir o processo de sucessão ecológica nas áreas em restauração.

Atualmente, têm-se buscado alternativas que possibilitem a redução dos custos de recuperação de áreas degradadas e o retorno dessas áreas a uma condição ecológica mais próxima da original, ou seja, a restauração ecológica (YOUNG et al., 2005; GANDOLFI et al., 2006). Nas últimas décadas, têm sido bastante difundida a utilização de sistemas agroflorestais (SAFs) como alternativa de restauração, pois estes, quando bem planejados, podem aproximar-se ecologicamente de comunidades florestais, em termos de diversidade, recuperando funções essenciais para a sustentabilidade, como a ciclagem de nutrientes e o acúmulo de biomassa, além de fornecerem alguma renda ou produção de subsistência ao agricultor (MACDICKEN; VERGARA, 1990).

Os SAFs fundamentam-se na sucessão natural de espécies (vegetais e animais) e na substituição ecofisiológica das espécies vegetais, buscando formar um sistema produtivo com estrutura, composição e funcionamento semelhantes à vegetação natural local, cuja dinâmica leva à regeneração das funções ambientais, à complexificação do ambiente e ao aumento da biodiversidade (SCHULTZ et al., 1994).

Estudos de avaliação em sistemas agroflorestais têm sido feitos com outras bases, a exemplo da produção e decomposição de serapilheira, do sequestro de carbono, da fitossociologia, da avaliação econômica e do aporte de nutrientes e da biomassa via serapilheira, como os trabalhos de Arato et al. (2003), Silveira et al. (2007), Rodrigues et al. (2007) e Froufe et al. (2011); no entanto, informações sobre o papel do banco de sementes como ferramenta de avaliação da sustentabilidade desses agroecossistemas ainda são escassos. Dessa forma, objetivou-se, por meio deste trabalho, avaliar a composição do banco de sementes como indicador de restauração, em quatro módulos de sistemas agroflorestais multiestratificados com diferentes idades, comparados a um remanescente florestal nativo, a fim de compreender se através do método de implantação e manejo desses sistemas foi permitido o armazenamento de sementes no banco do solo que promova a sustentabilidade das áreas onde os SAFs foram implantados.

\section{MATERIAL E MÉTODOS}

A área de estudo, denominada "Sítio da Mata”, localiza-se no Município de Amambai, no Sudoeste do Estado de Mato Grosso do Sul, fronteira com o Paraguai $\left(23^{\circ} 006^{\prime} \mathrm{S}\right.$ e $\left.55^{\circ} 014^{\prime} \mathrm{W}\right)$ a cerca de $480 \mathrm{~m}$ de altitude. Trata-se de uma propriedade agrícola com proposta de sistemas agroflorestais, empreendida pela iniciativa privada, especificamente pelo antropólogo e agricultor experimentador Paulo Pepe (in memoriam), cujo método de implantação e manejo dos SAFs se baseia no processo de sucessão natural proposto por Götsch (1995).

A formação florestal da região faz parte dos domínios da Floresta Atlântica e é classificada como Floresta Estacional Semidecidual (IBGE, 1992). O clima é considerado de transição entre o tropical e o subtropical e, segundo a classificação de Köppen, é do tipo Aw úmido com inverno seco e verão chuvoso.

Existem diversos módulos de SAFs implantados na propriedade com diferentes idades, arranjos de plantas e formas de implantação e manejo. Entre os módulos, efetuou-se a escolha de quatro, tidos na propriedade como principais e nomeados em função das espécies predominantes: Bananal (BAN/05 anos), Pomar (POM/16 anos), Macaubal (MAC/16 anos) e Erval (ERV/17 anos), nos quais a amostragem do banco de sementes foi realizada, bem como em uma área testemunha remanescente de mata nativa secundária (Figura 1). 


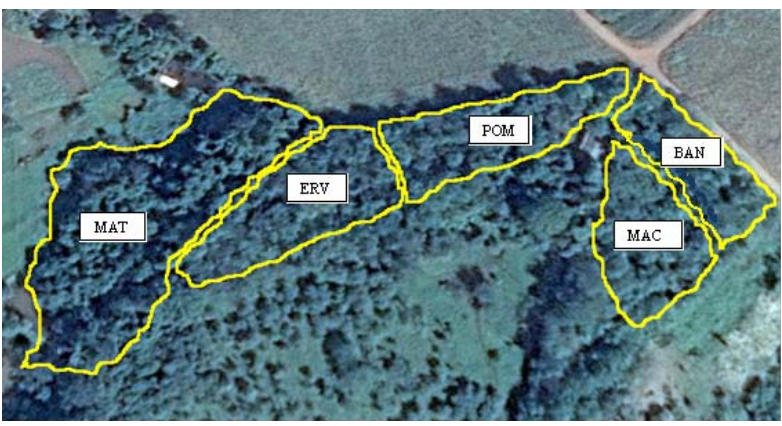

Figura 1 - Esquema baseado em imagem de satélite (Google Earth, 2013) com a disposição dos módulos agroflorestais e remanescente florestal nativo em Amambai, Mato Grosso do Sul, Brasil. BAN = Bananal, MAC $=$ Macaubal, $\mathrm{POM}=$ Pomar, ERV $=$ Erval e MAT = remanescente florestal.

Figure 1 - Scheme based on satellite image (Google Earth, 2013) with arrangement of agroforestry modules and remaining native forest in Amambai, MatoGrosso do Sul, Brasil.BAN = Bananal, MAC = Macaubal, $P O M=$ Pomar, $E R V=$ Ervaland $M A T=$ Remaining Forest.

De maneira geral, os SAFs estudados foram iniciados a partir do estabelecimento de culturas agrícolas associadas ao cultivo de espécies de rápido crescimento, utilizadas como fonte de biomassa e, gradualmente, foram introduzidas mudas e sementes de espécies arbóreas nativas, comuns nos remanescentes florestais da região, como: Enterolobium contortisiliquum (Vell.) Morong (Tamboril), Albizia niopoides (Spruce ex Benth.) Burkart (farinha-seca), Peltophorum dubium (Spreng.) Taub. (canafístula), Anadenathera peregrina (L.) Speg. (angico), Cordia trichotoma (Vell.) Arrab. Ex Steud (louro), Cinnamomum glaziovii (Mez) Kosterm. (canelacrespa), Ceiba speciosa (A.St.-Hil.) Ravenna (paineira), Guarea guidonia (L.) Sleumer (marinheiro) e Cedrela fissilis Vell. (cedro-rosa).

As coletas de solo foram realizadas em dezembro de 2012 e consistiram em 15 amostras distribuídas ao acaso em cada módulo de SAF e testemunha, correspondendo às repetições. Foi utilizado um gabarito de madeira de $20 \mathrm{~cm}$ x $20 \mathrm{~cm}$, e a profundidade avaliada foi de $5 \mathrm{~cm}$, escavada com o auxílio de espátula. A serapilheira não foi utilizada, pois, nesse caso, a intenção foi avaliar o depósito de sementes no solo.

Após a coleta, as amostras de cada área foram colocadas em sacos plásticos, etiquetadas e transportadas para a Faculdade de Ciências Biológicas e Ambientais da Universidade Federal da Grande Dourados FCBA/UFGD, acondicionadas em bandejas plásticas de 3 L e agrupadas, lado a lado, em estufa agrícola com revestimento em filme plástico, para evitar a contaminação por propágulos externos. A irrigação ocorreu diariamente por aspersão.

O monitoramento das plântulas foi realizado durante seis meses (180 dias), as quais cresceram até que atingissem tamanho que permitisse sua identificação, que foi realizada através de comparação com o acervo do herbário da Universidade Federal da Grande Dourados e com consulta à literatura especializada e a especialistas. O método utilizado para quantificação das sementes no solo foi o de emergência de plântulas ou germinação, conforme Gross (1990) e Brown (1992).

Foram calculados os índices de diversidade de Shannon (H') e de equabilidade de Pielou (J), para a comunidade do banco de sementes, bem como a densidade de sementes viáveis no solo de cada área. Para cada espécie, determinou-se a densidade relativa (DeRel), a frequência relativa (FrRel), a dominância relativa (DoRel) e o índice de valor de importância (IVI), com o cálculo realizado com o auxílio do programa FITOPAC (SHEPHERD, 1995).

As espécies amostradas foram classificadas conforme Angiosperm Phylogeny Group (APG II) (APG 2003) e a atualização taxonômica realizada mediante consulta ao banco de dados da Lista de Espécies da Flora do Brasil (LEFB, 2012).

\section{RESULTADOS}

Ao longo do período de avaliação, foram identificados 3.619 indivíduos nas cinco áreas de estudo, o equivalente a $1.206,3$ plantas $/ \mathrm{m}^{2}$, representadas por 63 espécies, distribuídas em 24 famílias. Desse total, 73\% foram de espécies herbáceas; 6,3\% de arbustivas; 14,2\% de arbóreas ;e 6,3\% de lianas. As famílias com maior riqueza de espécies foram Asteraceae, 14 espécies; Poaceae, 10; Euphorbiaceae, 6; e Solanaceae, 4. Das espécies arbóreas encontradas, sete são nativas: Trema micrantha (L.) Blume (296 indivíduos), Cecropia pachystachya Trécul (186), Solanum paniculatum L. (40), Macluria tinctoria (L.) D. Don ex Steud. (4), Solanum mauritianum Scop. (2), Croton urucurana Baill (1) e Alchornea triplinervia (Spreng.) Müll. Arg. (1) e duas exóticas, Carica papaya L. (11) e Leucena leucocephala (Lam.) de Wit. (6) (Tabela 1).

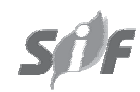

Revista Árvore, Viçosa-MG, v.38, n.6, p.1073-1083, 2014 
Tabela1 - Composição do banco de sementes de quatro módulos de Sistemas Agroflorestais (BAN, MAC, POM, ERV) e remanescente florestal nativo (MAT) no município de Amambai, MS. Número de plantas e valor de importância (VI) das espécies.

Table 1 - Composition of the bank of seeds of four modules of Agroforestry systems (BAN, MAC, POM e ERV) and remains native forest (MAT) in the municipality of Amambai, MS. Number of plants and importance value of species (VI).

\begin{tabular}{|c|c|c|c|c|c|c|c|}
\hline Famílias/Espécies & Nomes populares & $\begin{array}{l}\text { Forma } \\
\text { de vida }\end{array}$ & BAN & MAC & POM & ERV & MAT \\
\hline \multicolumn{8}{|l|}{ AMARANTHACEAE } \\
\hline $\begin{array}{l}\text { Alternantera tenella } \\
\text { Colla }\end{array}$ & Apaga-fogo & Erva & $16(12,05)$ & & & & \\
\hline $\begin{array}{l}\text { Amarantus hybridus var. } \\
\text { patulus (Betol.) Thel }\end{array}$ & Caruru & Erva & $4(5,34)$ & $8(4,84)$ & & & $2(1,84)$ \\
\hline $\begin{array}{l}\text { Chenopodium ambrosoides } \\
\text { L. }\end{array}$ & Ambrósia & Erva & $1(1,22)$ & & & & \\
\hline \multicolumn{8}{|l|}{ APIACEAE } \\
\hline Apium leptophyllum & Aipo-bravo & Erva & $1(1,04)$ & $1(1,03)$ & $47(21,96)$ & $28(6,92)$ & \\
\hline
\end{tabular}

(Pers.)

$1(1,08)$

Centella sp.

APOCYNACEAE

Schubertia grandiflora

Mart.

ARISTOLOCHIACEAE

Aristolochia triangularis

Cham. \& Achltdt

Cipó-mil-

Trepadeira

$5(2,94)$

ASTERACEAE

Ambrosia artemisiaefolia

L.

Agerantum conyzoides L.

Bidens pilosa L.

Trepadeira

$3(2,46)$

Conyza canadensis (L.)

Cronquist

Emilia sonchifolia (L.)

DC. Ex Wight

Artemijo

Erva

Picão-roxo

Picão-preto

Erva

\section{Erva}

Erva

$32,63)$

$3(1,21)$

$3(2,25)$

Buva

Erva

Pincel-de-

princesa

Galinsoga parviflora Cav.

Botão-de-

Gamochaeta pensylvanica

(Willd.) Cabrera

Gamochaeta purpurea

(L.) Cabrera

Mikania cordifolia (L.f.)

Willd

Macela

Erva

$2(2,65)$

$1(3,21)$

$3(2,25)$

Porophyllum ruderale

(Jacq.) Cass.

Siegesbeckia orientalis

L.

Sonchus oleraceus L.

Synedrellopsis grisebachii

Hieron \& Kuntze

Macela

Erva

ouro

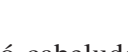

Erva

$66(28,39)$

$4(20,58)$

$21(11,71)$

$19(5,58)$

$1(0,91)$

Vernonia glabrata Less.

CANNABACEAE

Trema micrantha (L.)

Blume

Cipó-cabeludo Trepadeira

$21(8,88)$

$1(0,8)$

$30(11,54) 50(24,57)$

Arnica

Erva

$10(3,88)$

Brva

Serrália Erva

Agriãozinho-

Erva

$10(4,92)$

Assa-peixe

Arbusto

$29(9,89)$

$6(6,23)$

$12(5,8)$

$4(3,44)$

Assa-peixe

Árvore

$7(3,58)$

$9(6,76)$

$14(3,6)$

$1(1,03)$

$2(1,49)$

$2(2,35)$

Candiúva

Arvore

CARICACEAE

Carica papaya $\mathrm{L}$.

Mamão

Árvore

$2(6,29)$

$6(4,16)$

$2(1,29)$

$1(0,95)$

$1(0,75)$

$3(3,79) \quad 1(3,37) \quad 3(3,62)$

$2(7,83)$

$4(4,89)$

$20(8,97) \quad 26(15,09) \quad 113(22,45) \quad 137(48,6)$

Revista Árvore, Viçosa-MG, v.38, n.6, p.1073-1083, 2014 
Tabela 1 - Cont.

Table 1 - Cont

\begin{tabular}{|c|c|c|c|c|c|c|c|}
\hline Commelina comunis L. & Trapoeraba & Erva & $53(24,96)$ & $53(16,82)$ & $17(7,86)$ & $29(9,55)$ & $3(1,46)$ \\
\hline \multicolumn{8}{|l|}{ CYPERACEAE } \\
\hline Cyperus flavus (Vahl) & Junquinho & Erva & $71(45,17)$ & $27(20,73)$ & $18(16,52)$ & $24(11,13)$ & $8(6,36)$ \\
\hline $\begin{array}{l}\text { Nees. } \\
\text { Kyllinga odorata Vahl }\end{array}$ & Junquinho & Erva & $66(28,45)$ & $4(3,78)$ & $12(4,88)$ & & $2(2,09)$ \\
\hline \multicolumn{8}{|l|}{ EUPHORBIACEAE } \\
\hline $\begin{array}{l}\text { Alchornea triplinervia } \\
\text { (Spreng.) Müll. Arg. }\end{array}$ & Pau-óleo & Árvore & & & & & $1(1,09)$ \\
\hline $\begin{array}{l}\text { Chamaesyce prostrata } \\
\text { (Aiton) Small. }\end{array}$ & $\begin{array}{l}\text { Quebra-pedra- } \\
\text { rasteiro }\end{array}$ & Erva & $6(3,51)$ & & & & \\
\hline Croton urucurana Baill & Sangra-d’água & Árvore & $1(1,35)$ & & & & \\
\hline $\begin{array}{l}\text { Euphorbia heterophylla } \\
\text { L. }\end{array}$ & Leiteiro & Erva & $2(2,69)$ & $2(3,17)$ & & & $2(1,94)$ \\
\hline $\begin{array}{l}\text { Euphorbia hirta (L.) } \\
\text { Milisp. }\end{array}$ & $\begin{array}{l}\text { Erva-de- } \\
\text { Santa-Luzia }\end{array}$ & Erva & $28(7,52)$ & $110(29,03)$ & $81(26,94)$ & $267(94,86)$ & $77(25,44)$ \\
\hline Ricinus communis L. & Mamona & Arbusto & & $2(3,62)$ & & $1(2,48)$ & \\
\hline \multicolumn{8}{|l|}{ FABACEAE } \\
\hline $\begin{array}{l}\text { Leucaena leucocephala } \\
\text { (Lam.) de Wit. }\end{array}$ & Leucena & Árvore & & $6(5,75)$ & & & \\
\hline
\end{tabular}

(Lam.) de Wit.

\begin{tabular}{|c|c|c|c|c|c|c|c|}
\hline $\begin{array}{l}\text { Hyptis suaveolens (L.) } \\
\text { Poit. }\end{array}$ & Alfavacão & Arbusto & & & & $1(0,98)$ & \\
\hline Leonurus sibiricus L. & Rubim & Erva & $7(5,14)$ & $1(0,8)$ & $6(7,72)$ & $10(3,67)$ & \\
\hline \multicolumn{8}{|l|}{ MALVACEAE } \\
\hline Abelmoschus sp. & Quiabo & Arbusto & & $1(2,73)$ & & & \\
\hline Sida cordifolia L. & Guanxuma & Erva & $10(5,49)$ & $86(27,7)$ & $27(20,6)$ & $7(3,63)$ & \\
\hline Sida rhombifolia L. & Guanxuma & Erva & $1(1,26)$ & $4(3,1)$ & $1(1,19)$ & $4(2,01)$ & \\
\hline \multicolumn{8}{|l|}{ MORACEAE } \\
\hline $\begin{array}{l}\text { Maclura tinctoria (L.) } \\
\text { D.Don ex Steud. }\end{array}$ & Amora-brava & Árvore & & & & $3(1,51)$ & $1(0,92)$ \\
\hline \multicolumn{8}{|l|}{ ONAGRACEAE } \\
\hline $\begin{array}{l}\text { Ludwigia tomentosa } \\
\text { (Cambess.) H. Hara }\end{array}$ & Cruz-de-malta & Erva & & & & & $1(0,84)$ \\
\hline \multicolumn{8}{|l|}{ OXALIDACEAE } \\
\hline Oxalis corniculata L. & Trevo-azedo & Erva & $35(15,49)$ & $16(8,3)$ & $11(6,45)$ & $11(4,97)$ & $10(6,05)$ \\
\hline \multicolumn{8}{|l|}{ POACEAE } \\
\hline $\begin{array}{l}\text { Brachiaria decumbens } \\
\text { Stapf }\end{array}$ & Capim-braquiária & Erva & & & $2(2,78)$ & $1(0,9)$ & \\
\hline $\begin{array}{l}\text { Brachiaria plantaginea } \\
\text { (Link) Hitchc. }\end{array}$ & Capim-doce & Erva & & $12(4,68)$ & & $2(1,57)$ & $3(3)$ \\
\hline $\begin{array}{l}\text { Cynodon dactylon (L.) } \\
\text { Pers. }\end{array}$ & Grama-seda & Erva & $36(17,41)$ & $6(3,61)$ & $3(2,12)$ & $23(7,01)$ & $1(0,84)$ \\
\hline $\begin{array}{l}\text { Digitaria insularis (L.) } \\
\text { Fedde }\end{array}$ & Capim-amargoso & Erva & & & & $1(1,81)$ & \\
\hline $\begin{array}{l}\text { Echinochloa colona } \\
\text { (L.) Link }\end{array}$ & Capim-coloninho & Erva & & & $1(0,95)$ & & \\
\hline $\begin{array}{l}\text { Eleusine indica (L.) } \\
\text { Gaertn. }\end{array}$ & $\begin{array}{l}\text { Capim-pé- } \\
\text { de-galinha }\end{array}$ & Erva & & $4(4,11)$ & & $1(0,69)$ & $1(1,28)$ \\
\hline $\begin{array}{l}\text { Panicum maximum } \\
\text { Jacq. }\end{array}$ & Capim-colonião & Erva & $3(4,13)$ & & & & $1(1,8)$ \\
\hline
\end{tabular}


Tabela 1 - Cont.

Table 1 - Cont.

\begin{tabular}{|c|c|c|c|c|c|c|c|}
\hline $\begin{array}{l}\text { Paspalum notatum } \\
\text { Flüggé }\end{array}$ & $\begin{array}{l}\text { Capim-mato- } \\
\text { grosso }\end{array}$ & Erva & & & $1(2,17)$ & & \\
\hline Pennisetum purpureum & Capim-elefante & Erva & $1(3,07)$ & & & & \\
\hline $\begin{array}{l}\text { Setaria geniculata } \\
\text { P. Beauv. }\end{array}$ & $\begin{array}{c}\text { Capim-rabo- } \\
\text { de-raposa }\end{array}$ & Erva & $1(1,16)$ & & & $6(1,51)$ & \\
\hline PHYLLANTACEAE & & & & & & & \\
\hline $\begin{array}{l}\text { Phylantus corcovadensis } \\
\text { Muell. }\end{array}$ & Quebra-pedra & Erva & $124(34,2$ & $189(40)$ & $102(32,35)$ & $220(31,99)$ & $64(21,12)$ \\
\hline PORTULACACEAE & & & & & & & \\
\hline $\begin{array}{l}\text { Portulaca mucronata } \\
\text { Link }\end{array}$ & Onze-horas & Erva & & & $1(3,16)$ & $1(1,16)$ & \\
\hline $\begin{array}{l}\text { Talinum patens (Jacq.) } \\
\text { Willd }\end{array}$ & Maria-gorda & Erva & $4(3,52)$ & $14(12,44)$ & $32(22,38)$ & $16(6,6)$ & $13(10,76)$ \\
\hline RUBIACEAE & & & & & & & \\
\hline $\begin{array}{l}\text { Richardia brasiliensis } \\
\text { Gomes }\end{array}$ & Poaia-branca & Erva & & $1(0,8)$ & $1(0,98)$ & $11(3,28)$ & \\
\hline SCHROPHULARIACEAE & & & & & & & \\
\hline Veronica persica Poir. & Mentinha & Erva & $1(0,99)$ & $34(10)$ & $16(6,26)$ & $30(7,65)$ & $7(2,62)$ \\
\hline SMILACACEAE & & & & & & & \\
\hline Smilax L. & - & Trepadeira & & & & & $1(0,88)$ \\
\hline SOLANACEAE & & & & & & & \\
\hline Solanaceae sp. & - & Erva & & $8(5,95)$ & & $1(0,68)$ & \\
\hline $\begin{array}{l}\text { Solanum americanum } \\
\text { Mill. }\end{array}$ & Maria-pretinha & Erva & $2(1,18)$ & $4(3,8)$ & $3(3,01)$ & $8(3,94)$ & $18(11,72)$ \\
\hline $\begin{array}{l}\text { Solanum paniculatum } \\
\text { L. }\end{array}$ & Jurubeba & Árvore & & $1(0,96)$ & & $5(3,83)$ & $34(25,88)$ \\
\hline $\begin{array}{l}\text { Solanum mauritianum } \\
\text { Scop. }\end{array}$ & Fumo-bravo & Árvore & & & & $2(1,63)$ & \\
\hline URTICACEAE & & & & & & & \\
\hline $\begin{array}{l}\text { Cecropia pachystachya } \\
\text { Trécul }\end{array}$ & Embaúba & Árvore & $2(1,3)$ & $2(1,57)$ & $20(11,96)$ & $48(12,86)$ & $114(33,82)$ \\
\hline $\begin{array}{l}\text { Pilea microphylla (L.) } \\
\text { Liebm. }\end{array}$ & Brilhantina & Erva & $12(9,47)$ & $36(11,17)$ & $35(17,78)$ & $36(11,25)$ & $95(34,76)$ \\
\hline
\end{tabular}

A abundância de indivíduos de arbóreas nativas se apresentou de forma crescente de acordo com o aumento em idade das áreas, sendo no BAN (dois indivíduos), MAC (23), POM (46), ERV (171) e MAT (287). A riqueza de espécies de maneira geral variou de 31 (POM) a 38 (ERV e MAC) e o índice de diversidade $\left(\mathrm{H}^{\prime}\right)$, de 2,387 (MAT) a 2,760 (POM) (Tabela 2).

Com relação às formas de vida, é possível identificar nas áreas BAN e MAC que entre as 10 espécies com maior valor de importância predominam espécies herbáceas (Figura 2). Entretanto, nas três outras áreas (POM, ERV e MAT), entre as 10 espécies com maior valor de importância, encontram-se espécies arbóreas pioneiras, como Trema micrantha (candiúva), Cecropia pachystachya (embaúba) e Solanum paniculatum (jurubeba). No Pomar e Erval, destacam-se
Trema micrantha e Cecropia pachystachya, sendo estas com maiores valores de importância no Erval.

Tabela 2-Riqueza, abundância, índice de diversidade deShannon $\left(H^{\prime}\right)$ e equabilidade $(J)$ do banco de sementes emcada módulo de SAF e testemunha, no Município de Amambai, MS.

Table 2 - Richness, abundance, Shannon diversity index $\left(H^{\prime}\right)$ and equability $(\mathrm{J})$ of the seed bank in each module of agroforestry systems and control in the Municipality of Amambai, MS.

\begin{tabular}{lcccc}
\hline Áreas/Idade & $\begin{array}{c}\mathrm{N}^{\circ} \\
\text { espécies }\end{array}$ & $\begin{array}{c}\mathrm{N}^{\circ} \\
\text { indivíduos }\end{array}$ & $\left(\mathrm{H}^{\prime}\right)$ & $(\mathrm{J})$ \\
\hline Bananal/04 anos & 32 & 609 & 2,644 & 0,763 \\
Macaubal/16 anos & 38 & 786 & 2,707 & 0,744 \\
Pomar/16 anos & 31 & 562 & 2,76 & 0,804 \\
Erval/17 anos & 38 & 998 & 2,507 & 0,689 \\
Mata/indet. & 32 & 664 & 2,387 & 0,689 \\
\hline
\end{tabular}

Revista Árvore, Viçosa-MG, v.38, n.6, p.1073-1083, 2014 
Solanum paniculatum destaca-se entre as 10 espécies, com maior valor de importância apenas na área de Mata.
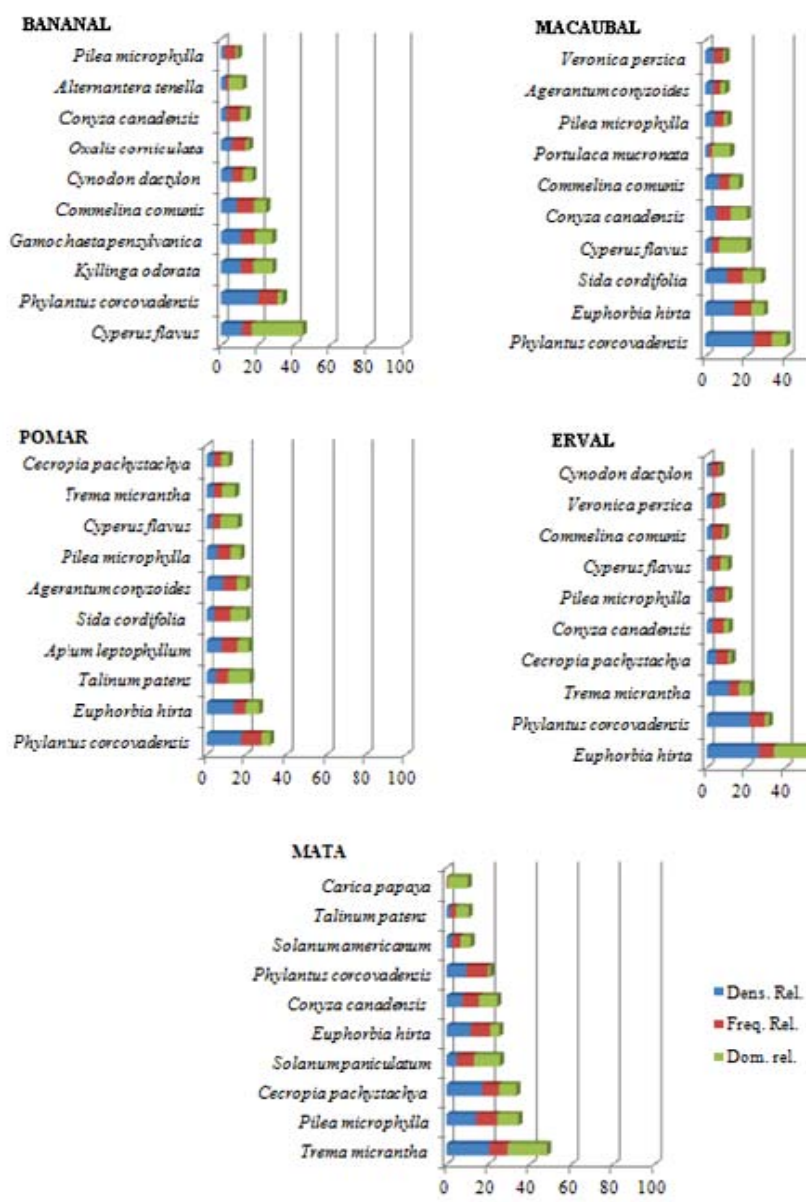

Figura 2 - Índice de valor de importância (IVI = densidade relativa + dominânciarelativa + frequência relativa) das 10 espécies de maior IVI nos SAFs e na testemunha (Mata), no Município de Amambai, MS.

Figure 2 -Importance value index (IVI= relative density + relative dominance + relative frequency) for the ten species with the highest IVI in the Agroforestry systems and control in the Municipality of Amambai, MS.

Tabela 3 - Densidade de sementes viáveis referentes a formas de vida emcada módulo de SAFs e testemunha, no Município de Amambai, MS

Table 3 - Density of viable seeds referring to life forms in each module of Agroforestry systems and control in the Municipality of Amambai, MS.

\begin{tabular}{|c|c|c|c|c|c|}
\hline Módulo/idade & $\begin{array}{c}\text { Espécies herbáceas } \\
\left(\text { sem. } / \mathrm{m}^{2}\right)\end{array}$ & $\begin{array}{c}\text { Espécies arbóreas } \\
\left(\text { sem. } / \mathrm{m}^{2}\right)\end{array}$ & $\begin{array}{c}\text { Espécies arbustivas } \\
\left(\mathrm{sem} . / \mathrm{m}^{2}\right)\end{array}$ & $\begin{array}{c}\text { Lianas } \\
\left(\text { sem. } / \mathrm{m}^{2}\right)\end{array}$ & $\begin{array}{l}\text { Densidade total } \\
\left(\text { sem. } / \mathrm{m}^{2}\right)\end{array}$ \\
\hline Bananal/04 anos & 1.010 & 5 & - & - & 1.015 \\
\hline Macaubal/16 anos & 1.250 & 48,3 & 5 & 6,6 & $1.309,90$ \\
\hline Pomar/16 anos & 850 & 80 & 6,6 & - & 936,6 \\
\hline Erval/17 anos & $1.361,60$ & 295 & 3,3 & 3,3 & $1.663,20$ \\
\hline Mata/indet. & 613,3 & 480 & - & 13,3 & $1.106,60$ \\
\hline
\end{tabular}

Revista Árvore, Viçosa-MG, v.38, n.6, p.1073-1083, 2014 
(Tabela 3). Houve predomínio de espécies herbáceas em todas as áreas, e os valores dessas espécies não apresentaram diminuição em relação ao aumento de idade das áreas. Ainda assim, os menores valores foram encontrados na área de Mata. Já a densidade de sementes de espécies arbóreas aumentou conforme o avanço em idade das áreas, variando de cinco sementes $/ \mathrm{m}^{2}$ no bananal a 480 sementes $/ \mathrm{m}^{2}$ na mata.

\section{DISCUSSÃO}

As causas das variações nos índices de diversidade $\left(\mathrm{H}^{\prime}\right)$ e valores de equabilidade $(\mathrm{J})$ podem ser observadas, comparando-se as áreas: Pomar (16 anos) apresentou o maior índice de diversidade, mesmo sendo a área com menor riqueza, e isso se deve ao seu maior valor de equabilidade. Erval (17 anos), por sua vez, mesmo sendo a área mais rica, no macaubal (16 anos), apresentou elevada dominância relativa da espécie herbácea Euphorbia hirta, tornando a área menos equitativa. Na área de mata foi obtido o menor índice de diversidade, diretamente relacionado com o baixo valor de equabilidade. Deve-se salientar, no entanto, que os índices de diversidade $\left(\mathrm{H}^{\prime}\right)$ e valores de equabilidade $\mathrm{J}$ ), nesse caso, pouco contribuem para uma leitura adequada da situação das áreas, pois o aumento nesses índices está relacionado com a distribuição e predominância de espécies herbáceas nos sistemas.

Vários estudos indicaram que ambientes mais abertos ou em estádio inicial de sucessão exibem alta riqueza de espécies, estando associada, principalmente, à presença de ervas (BAIDER et al., 2001; MARTINS; ENGEL, 2007; KUNS, 2011). Essas espécies, como todas as pioneiras, ocupam um sítio disponível à colonização e facilitam o estabelecimento de outras espécies, pois agem como abrigo para vetores de dispersão, melhoram as condições de fertilidade do solo pela produção de matéria orgânica e contribuem para a modificação do microclima (BAIDER et al., 1999). Dessa forma, mesmo com a especial importância que recebem as arbóreas em estudos sobre o banco de sementes não deve ser menosprezada a importância das ervas nas fases iniciais do processo de sucessão. A mesma importância possui as lianas, que, apesar de constituírem o grupo com menor número de espécies neste estudo, desempenham papel fundamental, principalmente, em florestas perturbadas, com o aporte de folhas para a produção de serapilheira (HORA et al., 2008).

Deve-se salientar que o remanescente florestal nativo utilizado como referência, apesar de apresentar maior abundância de plântulas de espécies arbóreas em relação aos outros sistemas estudados (287 indivíduos), é representado por apenas cinco espécies, excetuando-se um indivíduo exótico (mamão). A riqueza é baixa quando comparada a estudos como os de Nóbrega et al. (2009) e Baider et al. (1999), que trazem informações sobre o banco de sementes em remanescentes florestais secundários. Esse fato se deve, principalmente, à matriz onde toda a área de estudo está inserida, um contexto de monocultura extensiva, e à fragmentação florestal bastante acentuada, fato que dificulta consideravelmente a dispersão de propágulos. Deve-se atentar que a área MAC, mesmo possuindo a mesma idade da área POM (16 anos), está mais distante do remanescente florestal, fator que pode explicar a menor ocorrência de espécies arbóreas nessa área.

Vásques-Yanes (1998) discutiu a importante participação do gênero Trema em áreas desflorestadas, auxiliando o melhoramento das condições ambientais para que outras espécies mais avançadas na sucessão possam se estabelecer. Trema micrantha teve também destaque em número de indivíduos no trabalho de Gasparino et al. (2006). Vale ressaltar que essa espécie não foi utilizada ao longo da implantação dos SAFs e a sua presença se deve, principalmente, à sua eficiente dispersão por pássaros e grande longevidade das sementes no solo (VÁSQUES-YANES, 1998). A mesma importância possui o gênero Cecropia no banco permanente de sementes em florestas tropicais (VÁZQUEZ-YANES; GUEVARA-SADA, 1985).

O fato de não ter ocorrido diminuição na densidade de sementes herbáceas no solo, conforme o aumento em idade das áreas, pode ser explicado devido à dominância de algumas espécies herbáceas, conforme citado anteriormente, que ocasionam aumento desequilibrado na densidade de sementes herbáceas no solo.

Os resultados expõem claramente a evolução na regeneração de áreas antropizadas, conforme evidenciado por Baider et al. (2001), em estudo sobre o banco de sementes durante a regeneração da Floresta Atlântica no Sudeste do Brasil. Neste estudo, é exposta a

Revista Árvore, Viçosa-MG, v.38, n.6, p.1073-1083, 2014 
importância de se relacionarem os dados referentes à densidade de sementes viáveis no solo com as formas de vida das plantas, em que os resultados indicam que há tendência de redução na densidade de espécies herbáceas no banco de sementes e aumento de arbóreas com o avanço na sucessão em florestas secundárias. Martins et al. (2008) reforçaram que, como indicador de avaliação de restauração, o banco de sementes deve ser analisado quanto à distribuição da sua densidade entre espécies de diferentes formas de vida.

Visualizando os SAFs estudados como estratégia de restauração e considerando a baixa riqueza de espécies arbóreas representadas no banco de sementes, poderia ser proposto o enriquecimento das áreas com outras espécies vegetais, através dos diversos métodos de restauração, a exemplo da semeadura direta. No entanto, deve-se salientar que, além das espécies arbóreas pioneiras encontradas no banco de sementes, outras espécies arbóreas pertencentes a grupos sucessionais mais avançados e que geralmente não são representadas no banco de sementes estão presentes nas áreas. Estas foram introduzidas e se desenvolvem desde o início das implantações; portanto, além das sementes oriundas de remanescentes florestais vizinhos, os exemplares arbóreos dos próprios locais fornecerão sementes que contribuirão para a sustentabilidade das áreas.

\section{CONCLUSÃO}

A composição do banco de sementes nos sistemas agroflorestais estudados sugere o avanço sucessional dessas áreas de forma semelhante ao que ocorre em florestas em estádio secundário de sucessão, tendo em vista, principalmente, o incremento na densidade de sementes de espécies arbóreas ao longo dos anos, o que demonstra que os sistemas agroflorestais multiestratificados possuem potencial para restaurar áreas degradadas.

\section{AGRADECIMENTOS}

À família de Paulo Pepe (in memoriam), pela acolhida e oportunidade de estudo no Sítio da Mata; e à CAPES, pela bolsa de auxílio à pesquisa.

\section{REFERÊNCIAS}

APG II - ANGIOSPERM PHYLOGENY GROUP. An update of the angiosperm phylogeny group classification of the orders and families of flowering plants: APGII. Botanical Journal of the Linnean Society, v.141, p.399-436, 2003.

ARATO, H. D.; MARTINS, S. V.; FERRARI, S. H. S. Produção e decomposição de serapilheira em um sistema agroflorestal implantado para recuperação de área degradada em Viçosa-MG. Revista Árvore, v.27, n.5, p.715-721, 2003.

BAIDER, C.; TABARELLI, M.; MANTOVANI, W. O banco de sementes de um trecho de floresta Atlântica Montana (São Paulo). Revista Brasileira de Biologia, v.59, n.2, p.319-328, 1999.

BAIDER, C.; TABARELLI, M.; MANTOVANI, W. The soil seed bank during Atlantic Forest regeneration in Southeast Brazil. Revista Brasileira de Biologia, v.61, n.1, p.35-44, 2001.

BROWN, D. Estimating the composition of a forest seed bank: a comparison of the seed extraction and seedling emergence methods. Canadian Journal of Botany, v.70, n.8, p.1603-1612, 1992.

FENNER, M. Seed ecology. London: Chapman and Hall, 1985. 151p.

FROUFE, L. C. M.; SEOANE, C. E. S.

Levantamento fitossociológico comparativo entre um sistema agroflorestal multiestrato e capoeiras como ferramenta para execução da reserva legal. Pesquisa Florestal Brasileira, v.31, n.67, p.203-225, 2011.

FROUFE, L. C. M.; RACHWAL, M. F. G.; SEOANE, C. E. S. Potencial de sistemas agroflorestais multiestrata para sequestro de carbono em áreas de ocorrência de Floresta Atlântica. Pesquisa Florestal Brasileira, v.31, n.66, p.143-154, 2011.

GANDOLFI, S.; MARTINS, S. V.; RODRIGUES, R. R. Forest restoration. In: RODRIGUES, R. R.; MARTINS, S. V.; GANDOLFI, S. (Ed.) High diversity forest restoration in degraded areas: methods and projects in Brazil. New York: Nova Science Publishers, 2006. p.3-26.

GASPARINO, D.; MALAVASI, U. C.; MALAVASI, M. M.; SOUZA, I. Quantificação do banco de

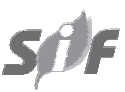

Revista Árvore, Viçosa-MG, v.38, n.6, p.1073-1083, 2014 
sementes sob diferentes usos do solo em área de domínio ciliar. Revista Árvore, v. 30, n. 1, p. 1-9, 2006.

GÖTSCH, E. O renascer da agricultura. Rio de Janeiro: AS-PTA, 1995. 22p.

GROSS, K. L. A. A comparison of methods for estimating seed numbers in the soil. Journal of Ecology, v.78, p.1079-1093, 1990.

HARPER, J. L. Population biology of plants. London: Academic Press, 1977. 892p.

HORA, R. C.; PRIMAVESI, O.; SOARES, J. J. Contribuição das folhas de lianas na produção de serapilheira em um fragmento de floresta estacional semidecidual em São Carlos, SP. Revista Brasileira de Botânica, v.31, n.2, p.277-285, 2008.

\section{INSTITUTO BRASILEIRO DE GEOGRAFIA E} ESTATÍSTICA - IBGE. Manual técnico da vegetação brasileira. Rio de Janeiro: 1992. 92p. (Série Manuais Técnicos em Geociências)

KUNS, S. H. O banco de sementes do solo e a regeneração natural em diferentes estádios sucessionais de floresta estacional semidecidual e de pastagem abandonada, reserva Mata do Paraíso, Viçosa, MG. 85f. Tese (Doutorado em Ciência Florestal) - Universidade Federal de Viçosa, Viçosa, MG, 2011.

\section{LEFB. Lista de Espécies da Flora do} Brasil. Disponível em: http://

floradobrasil.jbrj.gov.br/2012. Acesso em: 09 set. 2013.

MAC DICKEN, K. G.; VERGARA, N. T.

Agroforestry: classification and management. New York: Jonh Wiley \& Sons, 1990. 382p.

MARTINS, A. M.; ENGEL, V. L. Soil seed Banks in tropical forest fragments with different disturbance histories in southestern Brazil. Ecological Engineering, v.31, n.3, p.165-174, 2007.

MARTINS, S. V.; ALMEIDA, D. P.; FERNANDES, L. V.; RIBEIRO, T. M. Banco de sementes como indicador de restauração de uma área degradada por mineração de caulim em Brás Pires, MG.
Revista Árvore, v. 32, n. 6, p. 1081-1088, 2008.

NÓBREGA, A. M. F.; VALERI, S. V.; DE PAULA, R. C.; PAVANI. M. C. M. D.; S. A. SILVA, S. A. Banco de sementes de remanescentes naturais e de áreas reflorestadas em uma várzea do rio MogiGuaçu - SP. Revista Árvore, v. 33, n. 3, p. 403-411, 2009.

RODRIGUES, E. R.; CULLEN, L.; BELTRAME, T. P.; MOSCOGLIATO, A. V., SILVA, I. C. Avaliação econômica de sistemas agroflorestais implantados para recuperação de reserva legal no pontal do Paranapanema, São Paulo. Revista Árvore, v. 31, n. 5, p. 941-948, 2007.

RODRIGUES, R. R.; GANDOLFI, S. Restauração de florestas tropicais: subsídios para uma definição metodológica e indicadores de avaliação e monitoramento. In: DIAS, L. E.; MELLO. J. W. (Ed.). Recuperação de áreas degradadas. Viçosa, MG: Universidade Federal de Viçosa/ SOBRADE, 1998. p.203-215.

SCHULZ, B.; BECKER, B.; GÖTSCH, E. Indigenous knowledge in a "modern" sustainable agroflorestry system - a case study from eastern Brazil. Agroforestry Systems, v.25, n.1, p.59-69, 1994.

SHEPHERD, G. J. FITOPAC 1. Manual de usuário. Campinas: Departamento de Botânica, UNICAMP, 1995.

SILVEIRA, N. D.; PEREIRA, M. G.; POLIDORO, J. C.; TAVARES, S. R. L.; MELLO, R. B. Aporte de nutrientes e biomassa via serapilheira em sistemas agroflorestais em Paraty (RJ). Ciência Florestal, v. 17, n. 2, p. 129-136, 2007.

SIMPSON, R. L.; LECK, M. A.; PARKER, V. T. Seed banks: general concepts and methodological issues. In: LECK, M. A.; PARKER, V. T.; SIMPSON, R. L. Ecology of soil seed banks. San Diego: Academic Press, 1989. p.3-7.

VÁZQUEZ-YANES, C.; GUEVARA-SADA, S. Caracterización de los grupos ecológicos de árboles de las selva humeda. In: GOMEZ-POMPA, P.; AMO, S. D. (Ed.) Investigaciones sobre la regeneración de selvas altas em Veracruz, México. México: Alambra, 1985. Tomo II. p.76-78. 
VÁZQUEZ-YANES, C. Trema micrantha (L.) Blume (Ulmaceae): A promising neotropical tree for site amelioration of deforested land. Agroforestry Systems, v.40, n.1, p.97-104, 1998.
YOUNG, T. P.; PETERSEN, D. A.; CLARY, J. J. The ecology of restoration: historical links, emerging issues and unexplored realms. Ecology Letters, v.8, n.6, p.662-673, 2005. 
